\title{
Vers de nouveaux phénotypes et de nouvelles nosographies : de l'obésité aux maladies du tissu adipeux
}

\author{
Towards new phenotypes and nosography: from obesity to adipose tissue disorders
}

\author{
A. Basdevant $\cdot$ K. Clément $\cdot$ J.-M. Oppert \\ (C) Springer-Verlag France 2013
}

\begin{abstract}
Résumé L'obésité est classiquement définie à partir de seuils d'indice de masse corporels associés à une augmentation de la morbidité et de la mortalité. Cette approche est pertinente en termes d'analyse populationnelle mais elle connaît des limites au niveau individuel. Les progrès en épidémiologie, physiopathologie et clinique de l'obésité doivent orienter vers de nouveaux phénotypes et de nouvelles nosographies d'obésités, à inclure dans un cadre plus général, celui des maladies du tissu adipeux.
\end{abstract}

Mots clés Obésité $\cdot$ Phénotypes $\cdot$ Nosographie $\cdot$ Maladies du tissu adipeux

\begin{abstract}
The definition of obesity is based on thresholds of body mass index associated with an increase in morbimortality. This approach is relevant at a population level but it reaches some limits as far as the individual is concerned. Advances in epidemiology, physiopathology and clinics of obesity should lead to new phenotypes and nosography of obesity included in a general framework of adipose tissue disorders
\end{abstract}

Keywords Obesity - Adipose tissue disorders · Definition · Nosography

\section{Introduction}

Nous défendons ici l'idée qu'il est grand temps d'engager un questionnement exigeant sur la définition de l'obésité. Basée sur des seuils d'indice de masse corporelle, IMC, la définition classique de l'obésité a permis d'identifier les conséquences pathologiques de l'inflation de la masse grasse $a u$

A. Basdevant $(\varangle) \cdot$ K. Clément $\cdot$ J.-M. Oppert

Service de nutrition, Hôpital de la Pitié Salpêtrière,

Université Pierre et Marie Curie,

Institute of Cardiometabolism and Nutrition,

ICAN, Paris, France

e-mail : arnaud.basdevant@psl.aphp.fr niveau populationnel. Cette approche, à l'origine de l'émergence de l'obésité dans la médecine moderne, connaît actuellement des limites qui freinent la pratique clinique et la recherche biomédicale, et mettent en cause la pertinence de certaines actions de santé publique. Il faut dépasser la classification basée sur les seuils d'indice de masse corporelle pour aboutir à une nouvelle nosographie, regroupant dans un cadre plus global des " maladies du tissu adipeux » dont l'obésité au sens actuel représentera un sous-ensemble important mais non exclusif. Les arguments et propositions présentés dans cet article visent à animer le débat émergent sur cette question et ne prétendent pas présenter une solution aboutie.

Un rappel sur l'évolution des données épidémiologiques, médico-économiques et médicales ayant conduit à inscrire l'obésité parmi les situations pathologiques aidera à la mise en perspective des enjeux actuels autour de sa définition. Nous présenterons une série d'arguments pour faire évoluer cette définition avant de formuler quelques propositions.

\section{De Quetelet à la Metropolitan Life Insurance Company : émergence de l'obésité dans la médecine moderne}

Hippocrate, le premier, mentionne les inconvénients d'un excès de masse grasse pour la santé (« risque accru de mort subite ») et Gallien propose la première description raisonnée de la "polysarkia » [1]. Mais la notion de «norme pondérale » pour la santé apparaît au début du $19^{\mathrm{e}}$ siècle, notamment au travers des travaux d'Adolphe Quetelet. En charge d'analyser les caractéristiques anthropométriques de conscrits Ecossais, ce savant belge, médecin, statisticien, mathématicien, bute sur la mesure objective de l'adiposité que ne reflète pas le simple poids (il faut ajuster sur la taille), et recourt à une formule désormais célèbre, l'indice de masse corporelle (body mass index des anglo-saxons), conçue pour exprimer la corpulence en fonction de la taille (IMC : rapport du poids en $\mathrm{kg}$ sur la taille ${ }^{2}, \mathrm{~kg} / \mathrm{m}^{2}$ ). Il décrit la distribution 
« normale » de la corpulence et ses extrêmes « anormaux » $[2,3]$. C'est historiquement, la période de l'essor de la statistique des populations (Gauss) qui fonde l'approche positiviste de l'état pathologique selon laquelle la maladie est une dégradation d'un état parfait (dont on peut définir la norme, correspondant généralement à la moyenne ou à la médiane). Au $20^{\mathrm{e}}$ siècle, les compagnies d'assurance vont reprendre ces notions de références statistiques populationnelles pour définir des « facteurs de risque » et, à partir de là, moduler les niveaux de primes. Sont ainsi identifiés le tabagisme, l'hypercholestérolémie, l'hypertension artérielle ... et le poids idéal donc l'obésité. Vers 1942, les statisticiens de la Metropolitan Life Insurance Company établissent un lien entre longévité et le poids : la durée de vie est maximale quand le poids est maintenu durablement au niveau de celui de l'âge de 25 ans pour une taille donnée [4]. La perspective médico-économique est clairement inscrite dans la définition médicale de l'obésité. Quelques années plus tard cette compagnie d'assurance fixera ses tables de référence et l'obésité sera définie comme un excès de poids de $20 \%$ par rapport au poids souhaitable, niveau exposant à des dépenses de santé accrues, justifiant des primes supérieures. En 1985 un panel d'experts du NIH, National Institute of Health (USA) utilisera l'IMC et définira l'obésité comme un IMC supérieur à 27.8 chez l'homme et 27.3 chez la femme ce qui correspond environ aux valeurs de $20 \%$ d'excès par rapport aux poids idéal des assureurs [5]. En 1997, l'OMS (Organisation Mondiale de la Santé, WHO World Health Organisation) définit l'obésité par un IMC $>/=30$ et l'obésité sévère par un IMC $>40$ [6].

Après une marginalisation initiale au sein des facteurs de risque du fait de sa faible prévalence et donc de sa contribution modeste à la morbi-mortalité globale comparées à celle de l'hypertension, du tabac ou du diabète, l'obésité définie par l'IMC, va acquérir une notoriété médicale et publique grandissante à partir des années 1970-80. L'Amérique connaît alors une progression considérable de la prévalence de l'obésité considérée comme le marqueur pathologique d'une société de consommation, une maladie de la pléthore Nord-Américaine. Ce n'est qu'à partir des années 1990 que l'obésité étend son « territoire » au-delà des États-Unis pour atteindre la Grande Bretagne, progressivement le reste de l'Europe puis la quasi-totalité des pays du globe en particulier les pays émergents dont le Brésil, le Mexique, la Chine et une série d'autres pays dits " émergents » tels que les Emirats.

Les autorités de santé constatent que l'obésité a un impact médico-économique considérable en contribuant à la progression de maladies chroniques avant tout le diabète de type 2 (80\% des diabètes sont liés à l'obésité), l'hypertension artérielle et des maladies cardiovasculaires mais aussi certains cancers et des maladies respiratoires et articulaires sources de handicap. S'ajoute le retentissement psycholo- gique et social, le tout générant des coûts directs et indirects conséquents. Ces constats conduisent l'Organisation Mondiale de la Santé à publier un rapport «Obesity: the global epidemics» : l'obésité cesse d'être une simple mésaventure de la société fast food pour devenir une préoccupation de santé publique mondiale [6]. En France, en 2008, le Président de la Haute Autorité de Santé, HAS, déclare : «seuls les pays qui auront su maîtriser l'épidémie d'obésité pourront préserver leur système de protection sociale ». Dans cette même période, les progrès de la recherche vont donner à cette pathologie sa crédibilité scientifique. La science ouvre, au travers de différentes découvertes, des perspectives pharmacologiques qui ne peuvent laisser indifférents les acteurs économiques, les firmes pharmaceutiques. L'IMC fait donc son entrée dans la médecine au quotidien [7]. Il est au hitparade des propositions d'autoévaluation des sites internet de santé « Calculez votre IMC » «Etes-vous obèses 》 « Calculez votre poids normal».

\section{Les limites de l'IMC}

De ce rappel schématique sur l'émergence de l'obésité dans le champ de la médecine moderne, il faut retenir que la définition de l'obésité « se cherche » depuis 70 ans et ceci traduit un double questionnement : comment caractériser l'inflation adipeuse que l'on ne sait mesurer objectivement avec précision et quel est le seuil pertinent?

Tout d'abord comment caractériser l'inflation de la masse grasse?

Le diagnostic clinique d'obésité repose sur une formule, l'IMC, qui a une utilité certaine, on peut dire indiscutable pour les études populationnelles mais qui connaît, comme nous allons le voir, des limites au niveau individuel. L'inflation pathologique de la masse grasse est définie par un outil, l'IMC, qui ne mesure pas précisément ce qui est la base même de sa définition : le volume ou le poids de la masse grasse. C'est une situation singulière parmi les facteurs de risque. L'hyperglycémie est définie à partir d'un dosage dont la précision s'est affinée au fil du temps et qui mesure le taux de glucose dans le sang et rien d'autre. Idem pour le cholestérol. Il n'en est pas de même pour la masse grasse : nous cherchons à en évaluer les inconvénients mais nous ne disposons d'aucune méthode de mesure fiable pour la mesurer en routine qu'il s'agisse de la pratique clinique ou en épidémiologie chez un individu donné. Médecins, chercheurs, cliniciens ou épidémiologistes, décideurs, économistes, tous ceux qui s'intéressent à l'obésité ont recours à cette définition basée sur l'IMC et le grand public l'a intégrée. Si l'IMC a pris une place telle place dans la définition de l'obésité, c'est tout simplement qu'il n'existe pas de moyens simples de mesure de la masse grasse. Les méthodes biologiques sont en échec, les méthodes physiques 
comme l'impédancemétrie perdent de leur précision en cas d'inflation importante de la masse grasse, les moyens d'imagerie sont performants (absorptiométrie biphotonique, $\mathrm{RMN}$, etc) mais ils sont inaccessibles pour la pratique clinique [7-9]. Il faut bien admettre que le milieu médical et scientifique a trop longtemps ignoré ou toléré, comme le soulignent Prenctice et Jebb et Gema Fruhbeck, les limites et inconvénients de cette définition de l'obésité basée sur l'IMC [10,11]. Le rapport avantages/inconvénient de cette approche basée sur l'IMC a sans doute été positif pour identifier l'inflation de la masse grasse comme situation à impact médical mais actuellement les inconvénients l'emportent comme nous allons le voir, mais revenons aux limites de l'IMC.

Si l'IMC est un outil d'intérêt pour caractériser la corpulence des populations à l'aide de mesures simples et peu couteuses (une toise et une balance suffisent...encore fautil qu'elles soient justes !), sa valeur prédictive pour caractériser le degré d'inflation de la masse grasse au niveau individuel est faible. Pour un même IMC la composition corporelle est variable. Différents travaux montrent que la sensibilité de l'IMC pour évaluer la masse grasse n'est que de $50 \%$ [12-15]. Le cas des sportifs de haut niveau illustre ce point de manière caricaturale : certains athlètes ont un IMC qui dépasse le seuil définissant l'obésité en raison non d'une inflation de la masse grasse mais de celle de la masse musculaire. Au-delà de ces cas extrêmes, les variations de composition corporelle pour un même niveau d'IMC se constatent dans une population générale et entre populations. L'effet de l'âge est un premier exemple : à poids égal le sujet âgé a moins de masse maigre et plus de masse grasse. Les populations asiatiques ont plus de masse grasse que les populations caucasiennes pour un même IMC, la définition des seuils d'obésité est donc différente. Sans parler de situations pathologiques (œdèmes, sarcopénie rapide en contexte infectieux, etc) [9]. Le risque est clair : c'est celui de mauvaise classification des individus, le risque de sur- et de sousestimation des liens entre caractéristiques du tissu adipeux et santé. Ceci pose problème aussi bien au niveau populationnel qu'individuel. Il a été montré que la mesure de la masse grasse permettait de mieux classer les sujets à risque de complications liées à l'insulino-résistance et au diabète que ne le permet le seul IMC $[16,17]$.

Réciproquement, il faut évoquer la notion récente d'obésité " non pathogène », généralement mentionnée comme " healthy obesity », c'est-à-dire de situations où l'IMC entre dans les seuils dits anormaux sans qu'il existe de complications métaboliques ou vasculaires [18]. Cette notion de healthy obesity mériterait une analyse critique en soi car elle se limite le plus souvent aux aspects cardio-métaboliques et méconnaît bien d'autres impacts somatiques (par exemple les complications dites mécaniques telles que l'apnée du sommeil, inflammatoires telles que l'asthme, les maladies articulaires, hépatiques, etc), sans parler des impacts psychologiques et sociaux et des problèmes méthodologiques des études qui défendent ce concept. Néanmoins cette notion d'healthy obesity est un exemple frappant de problème de classification à partir de l'IMC [18]. S'ajoutent des notions récentes encore plus troublantes : l'effet paradoxal de l'obésité chez des patients en situation pathologique : les personnes obèses ont une meilleure survie que les personnes non obèses en cas d'insuffisance cardiaque, de dialyse, de réanimation. L'obésité peut dans certains cas être protectrice du risque de mortalité dans certaines situations pathologiques (que l'obésité elle-même a pu générer) [19-25].

Autre remise en cause récente, celle des seuils. De récentes analyses épidémiologiques ont questionné les seuils classiques, IMC 30 et IMC 40 . Une première remise en cause de ces seuils, nous l'avons évoqué, a résulté de données sur les populations asiatiques indiquant que pour un même IMC, la masse grasse était supérieure à celle des populations caucasiennes : il a été proposé d'abaisser les seuils d'IMC pour ces populations [10]. En sens inverse, une meta-analyse récente très médiatisée, publiée dans le New England Journal of Medicine indique que pour la mortalité les seuils d'IMC classiques ne se confirment pas et devraient être revu à la hausse dans les populations caucasiennes, la mortalité ne devenant réellement significative qu'à partir de 35 [26,27]. Mais les auteurs de l'article soulignaient que pour la morbidité la situation était différente : l'obésité et le surpoids définis classiquement restent associés à une série de morbidité.

Ces différents exemples illustrent notre difficulté actuelle à définir médicalement l'obésité au niveau individuel avec pour corolaire des questions sur les objectifs et stratégies thérapeutiques : Réduction de masse grasse ? Préservation de masse maigre ? Prévention et traitement des complications ? On le voit il n'y a pas simplement une question de classification mais également de cible thérapeutique. Ceci ne remet pas en cause l'apport de l'IMC dans l'identification des problèmes de santé liés à la corpulence pour les études épidémiologiques, faute de disposer d'autres outils, mais ceci incite à utiliser ce critère avec plus de nuance. L'utilisation normative de l'IMC pose de vrais problèmes en enfermant la réflexion médicale dans le cadre arbitraire, $\mathrm{du}$ « poids idéal théorique ». Il n'est pas licite d'infliger la même référence pondérale à chacun car pour un même IMC, les risques pour la santé différent grandement d'un individu à l'autre et d'une population à l'autre. Le regard social sur la corpulence contribue également à cette normalisation. L'obésité est une des rares situations «médicales » soumises au jugement individuel et collectif. Alors que le seuil de définition d'autres facteurs de risque comme l'hyperglycémie ou la pression artérielle n'est pas l'objet d'opinions profanes, chacun a son avis sur la corpulence. La définition individuelle de l'excès de poids s'inscrit dans un référentiel collectif. Chaque société construit sa norme qui influence une 
éventuelle décision de perdre du poids. Ainsi certaines personnes non obèses au sens médical, souhaitent atteindre un poids inférieur, pour se conformer à une image «idéale». A l'inverse, certains sujets obèses, qui entrent dans les critères médicaux de l'obésité, se sentent en parfait équilibre psychologique et physique. D'où une certaine confusion car milieu médical, individu, media, société entendent sous le terme « obésité » des notions diverses. L'utilisation d'une « norme médicale » (incertaine) contribue à une normalisation sociale de la corpulence qui expose à des dérives. Il en résulte un risque de surmédicalisation et de sous-médicalisation des problèmes de "surpoids ». La société s'empare d'une « norme » qui visait à définir des populations à risque pour l'appliquer de manière abusive et inadaptée à l'individu. L'incitation à restreindre durablement l'alimentation pour satisfaire cette " norme », peut dans certaines circonstances, en particulier dans des périodes de fragilité psychologique, notamment à l'adolescence, générer des désordres du comportement alimentaire, contribuer à la stigmatisation sociale, voire conduire à des inconvénients pour la santé au travers des fluctuations pondérales. Le point de vue médical ne peut s'abstraire de ces éléments et doit prendre en compte les différentes dimensions des définitions de l'obésité [7].

Plus de pertinence dans la définition des états pathologiques associés à des anomalies du tissu adipeux et une meilleure distinction entre les points de vue médicaux et sociétaux contribueraient à clarifier un domaine qui à bien des égards approche de la confusion.

\section{De l'IMC aux adiposités pathologiques : une (r)évolution inachevée}

Il est étonnant que la validité de l'IMC n'ait fait l'objet d'aussi peu de questionnement de la part des cliniciens en dehors du concept d'adiposité abdominale à risque que l'on doit à Jean Vague. Cette notion "d'obésité abdominale » seule remise en cause significative de la suprématie de l'IMC, a été un progrès considérable [28,29]. L'idée qu'indépendamment d'une inflation généralisée de la masse adipeuse (évaluée par l'IMC), son développement dans un territoire spécifique puisse être également pathogène, indépendamment du poids, remettait en cause radicalement une définition considérée comme acquise. La contribution de Jean Vague est majeure et sa découverte continue de générer des recherches et des développements cliniques. De même l'apport des études sur les lipoatrophies et dystrophies a été considérable en illustrant comment des anomalies segmentaires du tissu adipeux blanc peut générer des complications dont certaines se rapprochent de celles de l'obésité [30]. La contribution de Jean Vague n'était pas une simple évolution, c'était une véritable révolution remettant en cause profondément la validité de l'IMC. Cette révo- lution n'a pas été jusqu'au bout alors qu'elle ouvrait la voie, au dépassement des concepts classiques, à l'émergence des « adiposités segmentaires » pathogènes sans inflation globale de la masse grasse, à une réflexion en profondeur sur une nouvelle classification des maladies du tissu adipeux ou des tissus adipeux. Le fait qu'aucune évolution technologique n'ait permis au clinicien et à l'épidémiologiste de disposer d'une imagerie accessible des tissus adipeux a sans aucun doute contribué à figer la définition sur ses bases initiales. La mesure du tour de taille a donné une nouvelle dimension, mais il faut souligner que ce paramètre n'aborde pas la distinction entre graisse viscérale et graisse souscutanée,

L'IMC reste donc hégémonique et toute anomalie du tissu adipeux est définie au travers de son prisme. La pauvreté des classifications actuellement proposées pour définir l'obésité (OMS) ou pour identifier l'obésité dans le système d'information médicale est stupéfiante (Tableau 1). Si les pathologies du foie, du rein étaient restées à ce stade, c'est-à-dire à la mesure de la taille ou du volume d'un organe pour définir une maladie, l'hépatologie et la néphrologie seraient en mauvaise passe. Cette situation évoque la formule célèbre d'Abraham Maslow « si le seul outil que vous avez est un marteau vous aurez tendance à voir tout comme un clou ». L'IMC est l'unique outil de caractérisation des maladies des excès de poids et tout est jugé en fonction de son résultat. Et pourtant, les résultats de la recherche et tant de données cliniques depuis 50 ans, plaident pour une redéfinition des obésités tant sont hétérogènes les situations cliniques et physiopathologiques. Nous donnerons ici quelques éléments clés, non exhaustifs et non hiérarchiques, qui devraient contribuer à une refondation des maladies du tissu adipeux.

Premier élément, les évidences méritent parfois d'être mises en avant, le tissu adipeux est un organe qui assure des fonctions physiologiques qui contribuent au bien-être et à la santé : avant d'être potentiellement ou effectivement pathologique, il est essentiel à l'homéostasie. Un excès de ce tissu essentiel peut ou peut ne pas être pathologique et pathogène.

Deuxième élément, la diversité cellulaire des tissus adipeux. Les adipocytes représentent en nombre qu'un tiers de cellules du tissu, certes le tiers le plus visible mais pas le seul

Tableau 1 Classification des obésités dans l'International Statistical Classification of Diseases and Related Health Problems 10th Revision, ICD-10 « Obesity and other hyperalimentation »

Obesity due to excess calories E66

Drug induced obesity

E66-1

Extreme obesity + alveolar hypoventilation

E66-2

Other obesity

E66-8

Obesity unspecified: simple obesity NOS 
actif. Au sein même des adipocytes, l'hétérogénéité en termes de taille et de fonction est documentée. Le tissu adipeux n'est donc pas une simple collection d'adipocytes chargée de stocker des triglycérides à des fins énergétiques. En conséquence l'analyse anatomique et histologique du tissu adipeux devrait être le primum movens des processus d'analyse des situations cliniques. L'histopathologie du tissu adipeux est un élément primordial dans l'obésité. Les travaux pionniers sur la cellularité montrant la différence d'impact métabolique des obésités hypertrophiques et hyperplasiques, connaissent un regain d'intérêt depuis ces dernières années qui ont vu le plein développement de l'analyse anatomopathologique des tissus adipeux. Ainsi pour un même niveau de masse grasse, il existe une grande variabilité du volume adipocytaire. La notion d'une différence d'impact métabolique entre les obésités hypertrophiques et hyperplasiques devrait faire partie de nos phénotypes de routine tant les arguments sont solides dans ce domaine [31-33]. L'hypertrophie adipocytaire s'associe aussi à un état pro-inflammatoire aussi chez des femmes à IMC normal. A ce titre, cela pose la question du seuil adipocytaire ; existe-t-il seuil de volume adipocytaire au-delà duquel le risque inflammatoire et métabolique augmente ? Cette caractérisation de la cellularité et de son turn over s'est enrichie des données plus récentes sur l'inflammation, la fibrose, l'immunité des tissus adipeux [35-38], ainsi que de l'importance des réseaux vasculaires. Raréfactions mais gros vaisseaux inflammés et sénescents sont une marque de fabrique du tissu adipeux d'obèse.

Les conséquences cliniques de ces modifications cellulaires et tissulaires sont en cours d'identification et certaines ont d'ores et déjà un intérêt clinique. A titre d'exemple la fibrose du tissu adipeux sous-cutané qui peut affecter sur la plasticité et la rigidité tissulaire est un élément prédictif de réponse thérapeutique à la chirurgie bariatrique et est un marqueur de fluctuations pondérales itératives. Il est très étonnant de constater qu'un tissu aussi accessible ait pu être aussi longtemps méconnu histologiquement en clinique de l'obésité. S'ajoutent les extraordinaires avancées sur les liens et inter-conversion entre tissus adipeux blanc et tissus bruns [39]. Donc la caractérisation histopathologique, ne serait-ce que du tissu adipeux sous-cutané, devrait enrichir nos classifications cliniques de routine des anomalies du tissu adipeux. Celles-ci devraient également intégrer des marqueurs d'adiposité (leptine, adiponectine, insuline) et des paramètres tels que certaines adipokines ou d'autres marqueurs circulants d'inflammation, d'hypoxie ou de rétention de polluants [40-45]. Beaucoup de progrès sont encore nécessaires pour avancer dans ce domaine.

Il faut également revenir sur les modifications segmentaires, régionales du tissu adipeux qui indépendamment de l'inflation globale de la masse grasse, indépendamment du poids peuvent être à l'origine ou non d'effets pathogènes en étendant les concepts de Jean Vague.
Deux exemples parmi d'autres : le tissu adipeux péricardiaque et les tissus adipeux sous-cutanés abdominaux. Des modifications du tissu adipeux pericardique ou epicardique peuvent avoir des effets sur la fonction cardiaque indépendamment d'une inflation de la masse grasse [46]. Cela a été illustré récemment par l'identification du potentiel profibrotique du tissu graisseux autour de l'oreillette cardiaque élargissant les secrétions adipocytaires aux adipofibrokines [47]. Le tissu adipeux sous-cutané abdominal que l'on pensait homogène et protecteur vis-à-vis du risque vasculaire par rapport au tissu viscéral, est en réalité constitué de deux couches anatomiquement et physiologiquement, avec des conséquences bien différentes en termes de risque pour la santé. On pourrait également évoquer le rôle de la graisse péritumorale dans la physiopathologie de certains cancers.

La quantité de masse grasse ne suffit pas à définir une situation pathogène liée à l'adiposité. Il faut faire entrer l'imagerie dans le phénotypage des maladies du tissu graisseux. Ces éléments et bien d'autres plaident pour un démembrement cellulaire, tissulaire, anatomique et histopathologique des maladies du tissu adipeux, des tissus adipeux, de l'obésité, des obésités comme cela a été fait en hépatologie et en néphrologie depuis un siècle.

\section{Biologie, comportements, environnement : vers des phénotypes composites et dynamiques}

L'obésité ne peut être analysée selon un modèle pastorien classique, « un agent donne une maladie, l'éradication de cet agent supprime la maladie ». L'obésité, comme toutes les maladies chroniques, évolue, résulte d'interactions complexes entre des facteurs environnementaux, comportementaux et biologiques qui génèrent un processus évoluant en plusieurs phases : une phase préclinique sans manifestation observable mais où la prédisposition, biologique et non biologique, est présente, une phase d'initiation caractérisée par des perturbations fonctionnelles, généralement réversibles, éventuellement gênantes mais sans conséquence immédiate sur la santé, une phase d'aggravation et de passage à la chronicité avec apparition progressive de signes cliniques et de complications et une phase de résistance aux traitements (stade de maladie chronique) [7]. Cette notion de trajectoire doit être intégrée dans les phénotypes. De même, doivent être intégrés les éléments de comportements personnels, microenvironnement (« niche écologique » : habitudes familiales, culture, conditions de travail, école) et facteurs socioéconomiques (système alimentaire, urbanisation, etc) et plus globalement environnementaux. A titre d'exemple le rôle du microbiote, à l'interface du milieu intérieur et du milieu extérieur donc de l'environnement, est un élément qui apparaît intervenir de manière significative comme déterminant de l'obésité et/ou comme médiateur de ses conséquences. On 
se situe bien là à l'interface de l'individu et de son environnement, la lumière intestinale étant avec la peau et les alvéoles pulmonaires la zone d'échange la plus étendue avec le milieu extérieur, avec sa flore, forme de troisième partenaire de l'hôte et de l'environnement. Ce qui nous conduit à l'importance de la dimension contextuelle dans la définition des phénotypes d'obésité. Ceci n'est pas d'une actualité immédiate, car les recherches dans ce domaine sont encore récentes et évolutives mais cette dimension sera à l'évidence essentielle pour les futurs phénotypes $[48,49]$. A titre d'exemple parvenir à caractériser le comportement de l'individu dans son contexte de vie réelle, dans son quotidien, et ses relations avec des données géographiques au sens large fournira une troisième dimension du phénotypage (biologie, clinique, exposition).

Dans l'analyse des morbidités chez un patient donné, plusieurs éléments devraient être affinés. En premier lieu, en pratique clinique et de phénotypage, une approche exhaustive des complications doit être conduite, au-delà des rubriques classiques du risque vasculaire et métabolique. Il faut en réalité distinguer les complications de l'obésité en plusieurs rubriques selon les mécanismes prévalent : métaboliques, mécaniques, inflammatoires, immuns, oncologiques, psychologiques, sociaux, etc. Nous proposons dans le tableau 2 une classification des complications de l'obésité. Par ailleurs il faut démêler ce qui est causes ou conséquences. A titre d'exemple un trouble du sommeil peut être à l'origine d'une prise de poids ou être la conséquence d'une inflation majeure du tissu adipeux. De même pour la dépression. Des troubles du comportement alimentaire peuvent être à l'origine d'une obésité comme résulter de son traitement (par intolérance à la restriction alimentaire). Autre point important concernant les complications : ne pas mettre sous le même « chapeau » en termes de défaillance d'organe ou de fonction des situations relevant de mécanismes associés : prenons l'exemple de l'insuffisance cardiaque dont les composantes peuvent être «mécaniques », «nutritionnelles », respiratoires, etc. Il a été récemment proposé par un groupe de travail de l'EASO, European association for the study of obesity, de mieux distinguer les complications propres et spécifiques de l'excès de masse grasse (exemple stigmatisation, complications purement mécaniques dues à l'inflation adipeuse) de la contribution de l'obésité au développement des maladies chroniques. On entre là dans un débat espistémologique non anodin.

Le caractère essentiellement multidimensionnel et dynamique du processus qui conduit à l'inflation de la masse grasse n'est pas suffisamment pris en compte dans nos phénotypes de même que la distinction entre causes et conséquences de l'obésité. La trajectoire du patient, la dynamique de sa prise de poids sont des éléments clés pour lesquels il serait bien utile de disposer d'outil d'acquisition et de modélisation des informations. Il y a donc nécessité de grader les situations d'obésité non seulement en termes de dynamique temporelle du processus, mais d'impact sur la santé comme on le fait pour d'autres situations évolutives en clinique humaine (par exemple le « staging » des maladies du foie ou des situations cliniques en cancérologie). C'est ce que propose la classification d'Edmonton proposée par Sharma qui est une contribution innovante à la classification des obésités. Cette classification prend en compte l'importance de l'inflation de la masse grasse mais également les conséquences à la fois somatiques, médicales au sens habituel du terme mais également fonctionnelle ce qui rapproche de la vraie vie du point de vue du patient [54].

\section{De la théorie à la pratique : comment faire avancer la classification?}

Ce plaidoyer pour une refondation de la définition et de la classification des obésités et plus globalement des maladies des tissus adipeux ne sera convaincant que s'il parvient à des propositions pratiques pour les différents besoins que sont la pratique clinique, la recherche clinique, l'épidémiologie et à une nouvelle nosographie utilisable dans la classification internationale des maladies. Vaste programme loin d'être abouti qui suppose de reprendre des études épidémiologiques incluant de nouveaux paramètres sur lesquels il faudrait trouver un accord. C'est un travail collectif qui prendra du temps et de l'énergie mais qui nous paraît indispensable. C'est un travail qui devrait mobiliser l'ensemble de la communauté des épidémiologistes, chercheurs et cliniciens engagé dans ce domaine de la pathologie. Le chemin vers de nouveaux phénotypes et de nouvelles classifications sera long car il suppose la vérification de la pertinence d'une série de paramètres et d'hypothèses. Il sollicitera les nouvelles technologies de production (post-génomique) et de traitement de l'information. La contribution des bio-informaticiens est essentielle. C'est la condition pour aboutir à de nouveaux algorithmes de décision médicale tendant vers une médecine plus personnalisée.

On peut, pour amorcer le processus citer quelques éléments à introduire dans les différents cadres de pratique (épidémiologie, clinique, recherche). Pour la pratique clinique les données phénotypiques à enrichir concernent la mesure précise de la masse grasse par absorptiomètrie pour caractériser les " obésités 》 situées autour des valeurs seuil classiques, la cellularité du tissu graisseux par biopsie à l'aiguille, le staging évolutif et des complications. Pour la recherche clinique des phénotypes avancés composites et contextuelles doivent être recueillis dont la granularité est fonction des objectifs : données contextuelles (géographiques, informations écologiques), données comportementales dans la vraie vie (alimentaires et non alimentaires, notamment sur les addictions et l'impulsivité), données 
Tableau 2 Proposition de classification des conséquences de l'obésité.

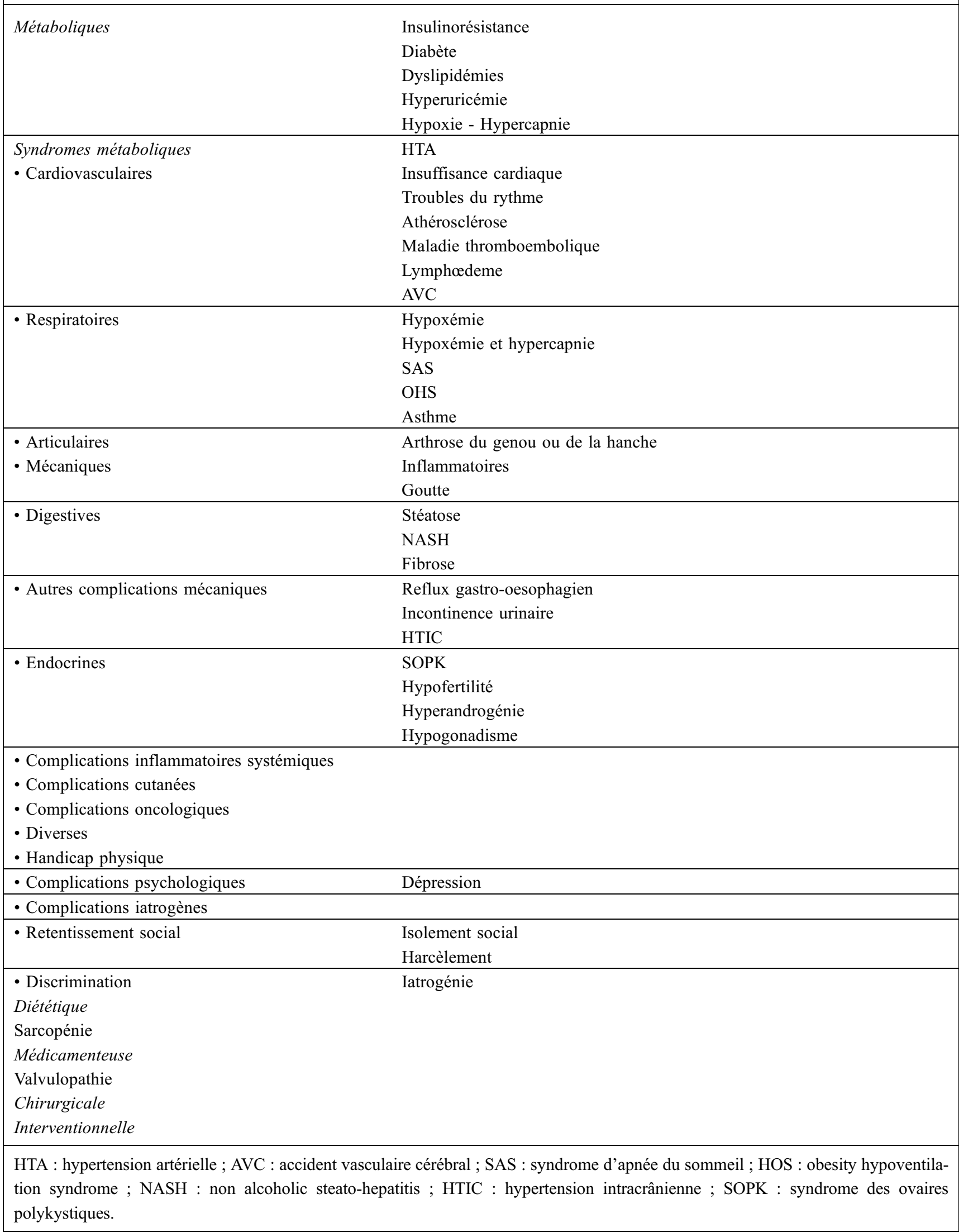


Tableau 3 Propositions de paramètres à inclure dans les phénotypes.

\begin{tabular}{|c|c|}
\hline Anthropométriques & $\begin{array}{l}\text { Poids, taille, tour de taille, tour de cuisse, tour de cou } \\
\text { Age du rebond pondéral } \\
\text { Masse grasse, masse maigre, masse osseuse (DEXA) } \\
\text { Rapport masse grasse sous-cutanée/viscérale } \\
\text { Rapport masse grasse/masse maigre } \\
\text { DER/masse maigre }\end{array}$ \\
\hline Cliniques & $\begin{array}{l}\text { Antécédents familiaux } \\
\text { Début : enfance adolescente adulte } \\
\text { Poids max poids minimum à l'âge adulte } \\
\text { Poids } 20 \text { ans } \\
\text { Nombre de fluctuations pondérales de plus de } 5 \text { ou de } 10 \mathrm{~kg} \\
\text { Évolution pondérale récente stable ascendante descendante }\end{array}$ \\
\hline Comportementaux & $\begin{array}{l}\text { Impulsivité } \\
\text { Restriction } \\
\text { Sédentarité } \\
\text { Activité physique } \\
\text { Apports caloriques estimés } \\
\text { Sous-estimation des apports }\end{array}$ \\
\hline Contextuels et sociaux & $\begin{array}{l}\text { - Statut professionnel } \\
\text { CSP } \\
\text { revenus } \\
\text { Indice de défavorisation } \\
\text { Trajectoire sociale } \\
\end{array}$ \\
\hline \multicolumn{2}{|l|}{ Complications cf. infra } \\
\hline Qualité de vie & $\begin{array}{l}\text { - Biologiques } \\
\text { Glycémie, EAL } \\
\text { Gamma GT transaminases } \\
\text { Gaz du sang } \\
\text { Marqeurs nutritionnels } \\
\text { CRP us } \\
\text { Leptine et adiponectine insulinémie } \\
\text { génétique } \\
\text { données transcriptomes ( ?) }\end{array}$ \\
\hline Histopathologiques sous-cutanées & $\begin{array}{l}\text { Taille cellulaire } \\
\text { Inflammation/immunité (marqueurs à définir) } \\
\text { Fibrose totale et péricellulaire }\end{array}$ \\
\hline Imagerie & $\begin{array}{l}\text { Tissu adipeux sous-cutané superficiel et profond tissus adipeux segmentaires } \\
\text { Stéatose hépatique } \\
\text { «rigidité tissulaire » (élastométrie ?) }\end{array}$ \\
\hline Explorations fonctionnelles & $\begin{array}{l}\text { EFR } \\
\text { Polygraphie ventilatoire } \\
\text { Capacité physique } \\
\text { Échographie cardiaque et artérielle } \\
\text { Fonction diastolique et systolique }\end{array}$ \\
\hline
\end{tabular}

DEXA : absorptiométrie bi-photonique ; DER : dépense énergétique de repos ; CSP : contrat de sécurisation professionnelle ; EAL : évaluation d'une anomalie lipidique ; YGT : gamma glutamyl transférases : CRP us : C reactive protein ultra-sensible : EFR : explorations fonctionnelles respiratoires. 


\begin{tabular}{|c|c|}
\hline $\begin{array}{l}\text { Atrophies du tissu } \\
\text { adipeux }\end{array}$ & $\begin{array}{l}\text { - Généralisée } \\
\text { Génétiques } \\
\text { Acquises } \\
\text { - Segmentaire } \\
\text { Génétiques } \\
\text { Acquises }\end{array}$ \\
\hline Hypotrophie & $\begin{array}{l}\text { Génétiques } \\
\text { Acquises }\end{array}$ \\
\hline \multicolumn{2}{|l|}{ Lipomes } \\
\hline Lipodystrophies & $\begin{array}{l}\text { - Partielles } \\
\text { - Généralisées } \\
\text { Génétiques } \\
\text { Acquises } \\
\text { Iatrogènes }\end{array}$ \\
\hline Hypertophie & $\begin{array}{l}\text { - Généralisée du tissu adipeux : obésité } \\
\text { - Segmentaire } \\
\text { Tronculaire } \\
\text { Viscérale } \\
\text { Sous-cutané } \\
\text { Superficielle } \\
\text { Profonde } \\
\text { Fémorale } \\
\text { Tissus ou organes } \\
\text { Mammaire } \\
\text { Cardiaque } \\
\text { Hépatique } \\
\text { Musculaire } \\
\text { Intestinale } \\
\text { Autre } \\
\text { Péritumorales }\end{array}$ \\
\hline $\begin{array}{l}\text { Pathologies } \\
\text { malignes }\end{array}$ & \\
\hline
\end{tabular}

biologiques issues des technologies de haut débit, données histologiques et d'imagerie. La difficulté est de traiter des informations de nature et de granularité extrêmement différentes. En guise d'incitation au débat, nous présentons dans les tableaux 2 à 4 une série de propositions que nous serons intéressés de voir argumentées, discutées, enrichies.

L'obésité est un concept issu de l'approche quantitative, d'une définition statistique d'un risque. L'enjeu clinique actuel est de retrouver une approche plus qualitative permettant des offres médicales « sur mesure ».

\section{Références}

1. Bray GA (1990) Obesity: historical development of scientific and cultural ideas. Int J Obes 14:909-26
2. Weigley ES (2000). Adolphe Quetelet. Am J Clin Nutr 71:853

3. Faerstein E, Winkelstein W Jr (2012) Adolphe quetelet: statistician and more. Epidemiology 23:762-3

4. Stavig GR, Leonard AR, Igra A, Felten P (1984) Indices of relative body weight and ideal weight charts. J Chronic Dis 37:255-62

5. WHO World Health Organization (1998) Obesity: preventing and managing the global epidemic. Report of a WHO Consultation on Obesity. Geneva, 1997 (WHO/NUT/NCD/98.1)

6. Clinical Guidelines on the Identification, Evaluation, and Treatment of Overweight and Obesity in Adults: The Evidence Report. National Institutes of Health (1998) Obes Res 6 Suppl 2:S51-209

7. Basdevant A, Bouillot JL, Clément K, et al (eds) (2011) Traité de Médecine et Chirurgie de l'obésité. Médecine Sciences Ed Lavoisier Paris

8. Thibault R, Genton L, Pichard C (2012) Body composition: why, when and for who? Clin Nutr 31:435-47

9. Browning LM, Mugridge O, Dixon AK, et al (2011) Measuring abdominal adipose tissue: comparison of simpler methods with MRI. Obes Facts 4:9-15

10. Prentice AM, Jebb SA (2001) Beyond body mass index. Obes Rev 2:141-7

11. Frühbeck $G$ (2008) Overview of adipose tissue and its role in obesity and metabolic disorders. Methods Mol Biol 456:1-22

12. Barreira TV, Harrington DM, Staiano AE, et al (2011) Body adiposity index, body mass index, and body fat in white and black adults. JAMA 306:828-30

13. Shah NR, Braverman ER (2012) Measuring Adiposity in Patients: The Utility of Body Mass Index (BMI), Percent Body Fat, and Leptin. PLoS One 7(4)

14. Müller MJ, Lagerpusch M, Enderle J, et al (2012) Beyond the body mass index: tracking body composition in the pathogenesis of obesity and the metabolic syndrome. Obes Rev 13 Suppl 2:6-13

15. Gómez-Ambrosi J, Silva C, Catalán V, et al (2012) Clinical usefulness of a new equation for estimating body fat. Diabetes Care 35:383-8

16. Gómez-Ambrosi J, Silva C, Galofré JC, et al (2011) Body adiposity and type 2diabetes: increased risk with a high body fat percentage even having a normal BMI. Obesity (Silver Spring) 19:1439-44

17. Frühbeck G, Toplak H, Woodward E, et al (2013) Obesity: The Gateway to Ill Health - an EASO Position Statement on a Rising Public Health,

18. Després JP (2012) What is "metabolically healthy obesity"? From epidemiology to pathophysiological insights. J Clin Endocrinol Metab 97:2283-5

19. Lavie CJ, De Schutter A, Milani RV (2013) Is there an obesity, overweight, or lean paradox in coronary heart disease? Getting to the 'fat' of the matter. Heart 7. [Epub ahead of print]

20. Tseng CH (2013) Obesity paradox: differential effects on cancer and noncancer mortality in patients with type 2 diabetes mellitus. Atherosclerosis 226:186-92

21. Florez H, Castillo-Florez S (2012) Beyond the obesity paradox in diabetes: fitness, fatness, and mortality. JAMA 8;308:619-20

22. Chrysant SG, Chrysant GS (2013) New insights into the true nature of the obesityparadox and the lower cardiovascular risk. J Am Soc Hypertens 7:85-94

23. Dixon JB, Lambert GW (2013) The obesity paradox--a reality that requires explanation and clinical interpretation. Atherosclerosis $226: 47-8$

24. Gielen S, Sandri M (2013) The obesity paradox - a scientific artifact? Int J Cardiol 162:140-2

25. Ferreira I, Stehouwer CD (2012) Obesity paradox or inappropriate study designs? Time for life-course epidemiology. J Hypertens 30:2271-5 
26. Flegal KM, Kit BK, Orpana H, Graubard BI (2013) Association of all-cause mortality with overweight and obesity using standard body mass index categories: a systematic review and metaanalysis. JAMA 309:71-82

27. Flegal KM, Ogden CL (2011). Childhood obesity: are we all speaking the same language? Adv Nutr 2:159S-66S

28. Vague J (1956) The degree of masculine diffentiation of obesities: a risk factor determining predisposition to diabetes, atherosclerosis, gout and uric acid. Am J Clin Nutr 4:20-34

29. Vague J (1996) Sexual differentiation. A determinant factor of the forms of obesity.1947. Obes Res 4:201-3

30. Capeau J, Magré J, Caron-Debarle M, et al (2010) Human lipodystrophies: genetic and acquired diseases of adipose tissue. Endocr Dev 19:1-20

31. Veilleux A, Caron-Jobin M, Noël S, et al (2011) Visceral adipocyte hypertrophy is associated with dyslipidemia independent of body composition and fat distribution in women. Diabetes 60:1504-11

32. Arner P (2010) Adipocyte turnover: relevance to human adipose tissue morphology. Diabetes 59:105-9

33. Rydén M (2013) Variations in the Size of the Major Omentum Are Primarily Determined by Fat Cell Number. J Clin Endocrinol Metab. Mar 29. [Epub ahead of print]

34. Frayn K, Bernard S, Spalding K, Arner P (2012) Adipocyte triglyceride turnover is independently associated with atherogenic dyslipidemia. J Am Heart Assoc Dec 1(6):e003467. doi: 10.1161/JAHA.112.003467

35. Langin D (2011) In and out: adipose tissue lipid turnover in obesity and dyslipidemia. Cell Metab 14:569-70

36. Tordjman J, Divoux A, Prifti E, et al (2012) Structural and inflammatory heterogeneity in subcutaneous adipose tissue: relation with liver histopathology in morbid obesity. J Hepatol 56:1152-8

37. Divoux A, Tordjman J, Lacasa D, et al (2010) Fibrosis in human adipose tissue: composition, distribution, and link with lipid metabolism and fat mass loss. Diabetes 59:2817-25

38. Ippoliti F, Canitano N, Businaro R (2013) Stress and obesity as risk factors in cardiovascular diseases: a neuroimmune perspective. J Neuroimmune Pharmacol 8:212-26
39. Langin D (2010) Recruitment of brown fat and conversion of white into brown adipocytes: strategies to fight the metabolic complications of obesity? Biochim Biophys Acta 1801:372-6

40. Márquez-Quiñones A, Mutch DM, Debard C, et al (2010) DiOGenes Project. Adipose tissue transcriptome reflects variations between subjects with continued weight loss and subjects regaining weight $6 \mathrm{mo}$ after caloric restriction independent of energy intake. Am J Clin Nutr 92:975-84

41. Bastard JP, Fève B (2012) The secretory face of the adipose cell: a tribute to two queens, leptin and adiponectin. Biochimie 94:2063-4

42. Mattu HS, Randeva HS (2013) Role of adipokines in cardiovascular disease. J Endocrinol 216:T1-36

43. Trayhurn P (2013) Hypoxia and adipose tissue function and dysfunction in obesity. Physiol Rev 93:1-21

44. Aron-Wisnewsky J, Minville C, Tordjman J, et al (2012) Chronic intermittent hypoxia is a major triggerfor non-alcoholic fatty liver disease in morbid obese. J Hepatol 56:225-33

45. La Merrill M, Emond C, Kim MJ, et al (2013) Toxicological function of adipose tissue: focus on persistent organic pollutants. Environ Health Perspect 121:162-9

46. Clément K, Basdevant A, Dutour A (2009) Weight of pericardial fat on coronaropathy. Arterioscler Thromb Vasc Biol 29:615-6

47. Venteclef N, Guglielmi V, Balse E, et al (2013) Human epicardial adipose tissue induces fibrosis of the atrial myocardium through the secretion ofadipo-fibrokines. Eur Heart J Mar 22. [Epub ahead of print]

48. Chaix B, Bean K, Daniel M, et al (2012) Associations of supermarket characteristics with weight status and body fat: a multilevel analysis of individuals within supermarkets (RECORD study). PLoS One. 7(4):e32908

49. Oppert JM, Charreire H (2012) The importance of the food and physical activity environments. Nestle Nutr Inst Workshop Ser 73:113-21

50. Padwal RS, Pajewski NM, Allison DB, Sharma AM (2011) Using the Edmonton obesity staging system to predict mortality in a population-representative cohort of people with overweight and obesity. CMAJ 183:E1059-66

51. Frühbeck G (2012) Obesity: Screening for the evident in obesity. Nat Rev Endocrinol 8:570-2 